\title{
Microinjection Technique to Relieve Neuropathic Pain by Infusion of Rapamycin into the Insular Cortex
}

\author{
Bae Hwan Lee ${ }^{1}$, Minjee Kwon ${ }^{2}$, Ran Won ${ }^{3}$ \\ ${ }^{1,2}$ Department of Physiology, Brain Korea 21 PLUS Project for Medical Science, Yonsei University College of Medicine, \\ 50-1 Yonsei-ro, Seodaemun-gu, Seoul 03722, Republic of Korea \\ bhlee@yuhs.ac; mjkwon486@yuhs.ac \\ ${ }^{3}$ Department of Biomedical Laboratory Science, Division of Health Sciences, Dongseo University, \\ 47 Jurye-ro, Sasang-gu, Busan 47011, Republic of Korea \\ wonran@gdsu.dongseo.ac.kr
}

\section{Extended Abstract}

The insular cortex (IC) of the brain has been conventionally considered the gustatory cortex [1]. However, recent study reported that the IC is associated with pain processing and modulation [2]. But the underlying molecular mechanisms remain unclear. The mammalian target of rapamycin (mTOR), a serine-threonine protein kinase, plays an important role in cell proliferation and differentiation by initiating mRNA translation in the nervous system [3]. Recently, mTOR and its downstream effectors have been shown to be involved in chronic pain such as neuropathic, inflammatory, and cancer-related pain in the spinal cord [4]. However, the role of the supraspinal mTORC1 signaling in neuropathic pain is still unknown. In the present study, we developed a microinjection technique to relieve neuropathic pain by infusion of rapamycin, an inhibitor of the mTOR, into the IC in an animal model of neuropathic pain. In addition, we investigated the role of mTOR signaling in the modulation of neuropathic pain.

Under pentobarbital anesthesia, adult male Sprague-Dawley rats were subjected for neuropathic surgery, and the development of neuropathic pain was evaluated by measuring mechanical allodynia. For the microinjection of rapamycin, 28-gauge guide cannulas were bilaterally implanted into the IC (AP: $+1.0 \mathrm{~mm}$, ML: $\pm 4.7 \mathrm{~mm}, \mathrm{DV}:-5.8 \mathrm{~mm}$ ). Hamilton syringes and PE-10 tubing with injection cannulas were used. Behavioral test and western blot analysis were performed on post-operative day 3 after infusion of rapamycin $(0.5 \mathrm{ul}, 600 \mathrm{nM})$ through the injection cannulas.

Nerve injury resulted in the development of mechanical allodynia. Microinjection of rapamycin into the IC reduced mechanical allodynia and the activation of mTOR signaling which is activated by nerve injury. Furthermore, rapamycin down-regulated the expression of postsynaptic density protein (PSD95) which is related with synaptic plasticity.

These findings suggest that inhibition of mTOR signaling in the IC may be a critical molecular mechanism that modulates neuropathic pain. Therefore, the microinjection of rapamycin into the IC may be a potential therapeutic treatment for the relief of chronic pain such as neuropathic pain.

This work was supported by the National Research Foundation (NRF) of Korea funded by the Ministry of Science, ICT, and Future Planning (NRF-2014R1A2A2A04004407).

\section{References}

[1] H. Ogawa, S. Ito, N. Murayama, and K. Hasegawa, . Taste area in granular and dysgranular insular cortices in the rat identified by stimulation of the entire oral cavity," Neurosci. Res., vol. 9, pp. 196-201, 1990.

[2] M. G. Liu, S. J. Kang, T. Y. Shi, K. Koga, M. M. Zhang, G. L. Collingridge, B. K. Kaang, and M. Zhuo, "Long-term potentiation of synaptic transmission in the adult mouse insular cortex: multielectrode array recordings," $J$. Neurophysiol., vol. 110, pp. 505-521, 2013.

[3] S. Huang and P. J. Houghton, "Targeting mTOR signaling for cancer therapy," Curr. Opin. Pharmacol., vol. 3, pp. 371377, 2003.

[4] S. M. Geranton, L. Jimenez-Diaz, C. Torsney, K. K. Tochiki, S. A. Stuart, J. L. Leith, B. M. Lumb, and S. P. Hunt, “A rapamycin-sensitive signaling pathway is essential for the full expression of persistent pain states," J. Neurosci., vol. 29, pp. 15017-15027, 2009. 